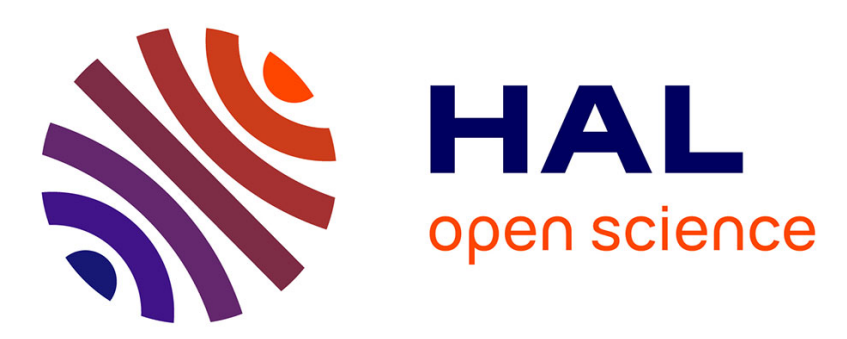

\title{
Arthrose, prévenir l'incapacité fonctionnelle et soulager la douleur
}

\author{
Nicolas Clere
}

\section{To cite this version:}

Nicolas Clere. Arthrose, prévenir l'incapacité fonctionnelle et soulager la douleur. Actualités Pharmaceutiques, 2019, 58, pp.45 - 47. 10.1016/j.actpha.2018.11.008 . hal-03485623

\section{HAL Id: hal-03485623 \\ https://hal.science/hal-03485623}

Submitted on 20 Dec 2021

HAL is a multi-disciplinary open access archive for the deposit and dissemination of scientific research documents, whether they are published or not. The documents may come from teaching and research institutions in France or abroad, or from public or private research centers.
L'archive ouverte pluridisciplinaire HAL, est destinée au dépôt et à la diffusion de documents scientifiques de niveau recherche, publiés ou non, émanant des établissements d'enseignement et de recherche français ou étrangers, des laboratoires publics ou privés.

\section{다)(1) $(5$}

Distributed under a Creative Commons Attribution - NonCommercial| 4.0 International 
Dochead pratique

Sous-dochead conseil

\section{Arthrose, prévenir l'incapacité fonctionnelle et soulager la douleur}

\section{Nicolas Clere}

Maître de conférences

Faculté de santé, Département pharmacie, Université d'Angers, 16 boulevard Daviers, 49045 Angers, France

Adresse e-mail : nicolas.clere@univ-angers.fr (N. Clere).

\section{Résumé}

L'arthrose est la maladie articulaire la plus fréquente. Le vieillissement de la population, l'hérédité, les anomalies du métabolisme ainsi que des pathologies telles que l'obésité et les traumatismes ou microtraumatismes répétés sont impliqués. Les principaux objectifs de prise en charge sont le soulagement de la douleur et le maintien de la fonction articulaire.

(C) 2018

Mots clés - articulation ; arthrose ; douleur ; inflammation

Summary à venir

(C) 2018

Keywords à venir

L'arthrose touche environ $65 \%$ des Français et $80 \%$ des Américains de plus de 65 ans [1]. II s'agit de la première cause d'incapacité fonctionnelle après 40 ans [2].

Cette pathologie se définit comme une affection dégénérative caractérisée par un ensemble de désordres aboutissant à un défaut structural et fonctionnel d'une ou plusieurs articulations. $\mathrm{Si}$, pendant de nombreuses années, il a été avancé que seuls les cartilages étaient affectés, il est aujourd'hui admis que l'arthrose constitue une atteinte de l'ensemble de l'articulation. La dégradation des cartilages est lente et s'accompagne de modifications morphologiques qui peuvent être recherchés à l'examen radiographique en vue d'établir un diagnostic. 


\section{T1 Processus de dégradation du cartilage}

TEG1 À l'état physiologique, le cartilage est un tissu dont les cellules se divisent relativement peu tandis que les fibres de collagène qu'il contient ne se renouvellent quasiment pas [3]. Les chondrocytes qui le composent sont des cellules hautement différenciées, responsables de I'homéostasie de la matrice : ils assurent la synthèse et la dégradation de tous ses composants grâce, notamment, à leurs propriétés anaboliques et cataboliques.

TEG1 Lorsqu'il devient arthrosique, le cartilage présente des cellules de morphologie et de phénotype modifiés, un processus qui peut aboutir à leur mort par apoptose. Par ailleurs, la quantité d'eau qu'il contient diminue considérablement : "l'effet éponge" du tissu disparaît, ce qui explique qu'il perd certaines de ses fonctions biomécaniques. L'ensemble de ces modifications entraîne peu à peu la diminution de ses capacités de résistance face aux forces auxquelles il est soumis. Enfin, la membrane synoviale est le siège d'une inflammation plus ou moins importante qui peut se traduire cliniquement par la présence d'un épanchement intra-articulaire.

TEG1 Sur le plan clinique, si les signes généraux ne sont pas rapportés, l'arthrose se manifeste par une douleur mécanique dont l'intensité est très variable. Celle-ci augmente en fonction des contraintes subies par l'articulation, se caractérise par une prédominance diurne et maximale le soir, et s'atténue avec le repos. Elle peut gêner l'endormissement mais ne réveille pas dans la seconde moitié de la nuit. Parfois, il peut être rapporté une raideur matinale qui cède rapidement, en moins de 15 minutes, même en l'absence de traitement. La limitation des mobilités est modérée tandis que la mobilisation déclenche des douleurs qui restent acceptables. Dans les formes compliquées, une réaction synoviale, secondaire à une altération du cartilage, est parfois observée.

\section{T1 Prévention}

TEG1 Les articulations doivent être protégées et ménagées tout au long de la vie. Ainsi, dans le cadre d'une activité sportive, même légère, l'échauffement et l'acquisition de bons gestes techniques sont indispensables pour éviter de les surcharger. Toutefois, l'exercice physique est bénéfique en vue de limiter le risque d'arthrose comme son évolution. Dans cette optique, il est conseillé de poursuivre ou débuter une activité d'intensité modérée. En cas d'articulation douloureuse ou enflée à l'issue de la séance, l'application d'un coussin thermique froid (Actipoche ${ }^{\circledR}$ chaud-froid, Nexcare $3 \mathrm{~m}$ Coldhot $^{\circledR}$, coussin thermique ${ }^{\circledR}$ froid/chaud ${ }^{\circledR}$ Medical Fournier) pendant au moins 20 minutes est conseillée.

TEG1 L'obésité joue un rôle non négligeable parmi les facteurs favorisant l'arthrose. Il doit être conseillé aux patients concernés de perdre du poids afin de réduire les risques d'atteinte des hanches et des genoux.

TEG1 Une rééducation à la marche peut être proposée, en accord avec un kinésithérapeute.

En effet, certaines personnes plantent leurs talons dans le sol, soumettant ainsi leurs genoux et leurs hanches à de forts impacts. L'objectif, pour ces patients, sera d'adopter une marche souple limitant 
les risques d'arthrose.

TEG1 Bien choisir ses chaussures de sport est primordial. De bonnes semelles et des talons qui amortissent les chocs sont les critères essentiels.

TEG1 Enfin, le surmenage et les efforts intenses (jardinage, bricolage sur plusieurs jours) sont proscrits en cas de crise arthrosique.

\section{T1 Prise en charge médicamenteuse}

TEG1 Les premières mesures sont l'information des patients et la dispensation de conseils hygiénodiététiques (perte de poids ou mise en place d'une activité physique modérée).

TEG1 Le paracétamol constitue l'antalgique de première intention dans le traitement de la douleur arthrosique en raison d'une très bonne efficacité et d'un faible risque iatrogène [4]. La posologie conseillée est de 1 gramme par prise, sans dépasser 3 grammes par jour ${ }^{1}$. Les effets indésirables sont extrêmement rares : hypersensibilité, œdème de Quincke, érythème, urticaire, rash cutané...

TEG1 Les anti-inflammatoires non stéroïdiens (AINS) tels que l'ibuprofène peuvent être proposés en seconde intention, en particulier chez les patients ne présentant pas de risque d'ulcère gastrique ou d'hémorragie digestive, qui constituent une contre-indication tout comme la grossesse (encadré 1) [5]. Certains AINS (diclofénac [Voltarenactigo ${ }^{\circledR}$ ], ibuprofène [Cliptol ${ }^{\circledR}$, Ibutop ${ }^{\circledR}$ ]) pour application locale peuvent soulager les douleurs siégeant au niveau des petites articulations (doigts...). Ces gels étant susceptibles d'entraîner des réactions de photosensibilité, les patients sont invités à couvrir les zones traitées durant le traitement mais également pendant les 15 jours suivant son arrêt.

\section{Encadré 1. Risque iatrogène des AINS}

L'Agence nationale de sécurité du médicament et des produits de santé (ANSM) a rappelé, dans une note de janvier 2017, que les anti-inflammatoires non stéroïdiens (AINS) sont formellement contreindiqués chez les femmes enceintes au-delà de la $24^{\mathrm{e}}$ semaine d'aménorrhée en raison d'un risque grave, pour le fœtus, d'atteintes rénales et cardio-pulmonaires pouvant être irréversibles, voire mortelles.

Sur 2 colonnes (une seule colonne de texte à l'intérieur)

TEG1 Les anti-arthrosiques symptomatiques d'action lente (chondroïtine sulfate, glucosamine, insaponifiables d'avocat et de soja) sont utilisés en traitement de fond. Le but est de soulager les symptômes, notamment en cas d'arthrose du genou ou de la hanche, mais aussi de limiter la prise d'antalgiques et ralentir la dégradation de l'articulation. En aucun cas, ces molécules ne présentent d'action immédiate en cas de poussées dont elles permettent, en revanche, d'espacer la survenue. 
En dépit de données scientifiques montrant une activité supérieure au placebo, la HAS a estimé que leur service médical rendu (SMR) est insuffisant pour permettre leur remboursement [4].

Les patients souhaitant les utiliser doivent consulter leur médecin traitant afin d'évaluer avec lui les avantages et les inconvénients.

\section{T1 Prise en charge alternative}

Différentes plantes peuvent être conseillées en complément (ou non) des antalgiques. Les huiles essentielles (HE) et les compléments alimentaires compléteront le conseil officinal.

TEG1 Les racines d'Harpagophytum procumbens, riches en iridoïdes, sont utilisées dans la prise en charge des douleurs rhumatismales quelle que soit leur localisation. L'harpagophytum présente des propriétés anti-inflammatoires, analgésiques et spasmolytiques qui contribuent à améliorer la mobilité et à soulager les symptômes douloureux. Cette plante peut être employée seule ou en association à d'autres thérapeutiques. Contre-indiqué chez les personnes souffrant de pathologies gastriques, cardiovasculaires et chez la femme enceinte, l'harpagophytum s'administre sous forme de gélules, à raison d'une trois fois par jour.

TEG1 Les feuilles du cassis (Ribes nigrum) sont riches en flavonoïdes, tanins et vitamine C. Leurs propriétés anti-inflammatoires sont susceptibles de lutter contre les manifestations articulaires douloureuses, notamment au niveau du genou.

TEG1 Les feuilles et l'écorce de saule blanc (Salix $\mathbf{a l b a}$ ), en raison de leur forte teneur en dérivés salicylés, ont des propriétés antipyrétiques et anti-inflammatoires, sans présenter d'effets indésirables gastriques. Elles peuvent être utilisées en association à l'harpagophytum ou aux feuilles de cassis.

TEG1 Les sommités fleuries de reine-des-prés (Filipendula ulmaria) contiennent des flavonoïdes et des dérivés salicylés dont les propriétés anti-inflammatoires et analgésiques sont intéressantes dans les manifestations articulaires douloureuses, et permettent aussi de réduire la consommation d'AINS. Il convient de prendre en compte les effets diurétiques de cette drogue, en particulier chez les personnes à risque, justifiant sa prise de préférence le matin. Ses propriétés seront potentialisées en association à l'harpagophytum dans la prise en charge de l'arthrose.

TEG1 Diverses HE associées dans un mélange d'huile végétale peuvent être appliquées sur les articulations douloureuses. Ainsi, celles de Gaulthérie (anti-inflammatoire), Menthe poivrée (antalgique), Lavande aspic (antalgique) et Ylang-ylang (anti-inflammatoire) peuvent être conseillées. Toutefois, les HE de Menthe poivrée et de Lavande aspic sont formellement contre-indiquées chez la femme enceinte et allaitante, ainsi que chez l'enfant de moins de 7 ans et la personne asthmatique ou épileptique.

TEG1 Du côté des compléments alimentaires, les oméga 3 présenteraient des propriétés antiinflammatoires protectrices des articulations. Consommés en début de journées, ces acides gras soulageraient aussi les raideurs matinales. En dépit d'un goût désagréable, ces huiles sont bien 
tolérées. Quelques nausées et épistaxis peuvent survenir en cas de surdosage, ce qui doit conduire un patient sous anticoagulant à réaliser un strict suivi de l'international normalized ratio (INR).

TEG1 Les vitamines $\mathbf{C}$ et $\mathbf{E}$ permettraient de limiter la progression des lésions cartilagineuses, notamment en raison de leurs effets antioxydants.

TEG1 Le cuivre est un oligo-élément qui accélérerait la reconstitution du cartilage en diminuant l'inflammation. Il peut être conseillé en cas de rhumatismes chroniques et déformants chez les personnes actives, voire de poussées inflammatoires.

TEG1 Les traitements homéopathiques sont difficiles à mettre en place car ils doivent être adaptés à chaque patient. Toutefois, il est possible de recommander :

- Rhus toxicodendron $9 \mathrm{CH}$ (cinq granules quatre fois par jour) si les douleurs sont aggravées par le repos et améliorées par le mouvement lent et progressif ;

- Radium bromatum $9 \mathrm{CH}$ (cinq granules trois fois par jour) en cas de recrudescences nocturnes ;

- Actea spicata $5 \mathrm{CH}$ associé à Polygonum aviculare $5 \mathrm{CH}$ (cinq granules de chaque au coucher) contre les raideurs et douleurs au lever dans l'arthrose digitale ;

- Arnica montana $9 \mathrm{CH}$ associé à Bryonia alba $9 \mathrm{CH}$ (cinq granules de chaque trois fois par jour pendant 10 jours) en cas d'hydarthrose inflammatoire.

- Arthrodrainol ${ }^{\circledR}$ (20 gouttes trois fois par jour), Rhus toxicodendron complexe $n^{\circ} 80^{\circledR}$ (20 gouttes trois fois par jour ou toutes les heures en cas de douleurs aiguës), Urarthone ${ }^{\circledR}$ (une cuillère à soupe deux fois par jour dans une infusion chaude) associent différentes souches homéopathiques présentant un intérêt thérapeutique dans la prise en charge de l'arthrose.

TEG1 Certains soins locaux à base de plantes pourront également être conseillés :

- des bains ou lotions de romarin ;

- des baumes chauffants tel Dolpic ${ }^{\circledR}$ avec ou sans capsaïcine ;

- des produits réfrigérants comme Phytalgic ${ }^{\circledR}$ gel, composé d'isopropoanol qui diminue la température locale à la surface de la peau et procure un effet apaisant qui sera optimisé par un léger massage. Le menthol participe également à cette action. Avec l'eucalyptol, il apporte une sensation de fraîcheur.

\section{Points à retenir}

- L'arthrose est une affection dégénérative qui entraîne un défaut structural et fonctionnel d'une ou plusieurs articulations. 
- Elle se manifeste par une douleur à prédominance diurne, maximale le soir et qui s'atténue avec le repos, et une limitation des mobilités.

- Les articulations doivent être ménagées tandis que l'exercice physique d'intensité modérée et une perte de poids en cas d'obésité seront conseillés.

- La prise en charge repose sur le paracétamol en première intention, les anti-inflammatoires non stéroïdiens en deuxième intention, voire sur les w symptomatiques d'action lente et des thérapeutiques alternatives.

Sur 2 colonnes (une seule colonne de texte à l'intérieur)

\section{T1 Conclusion}

En complément du conseil officinal classique, des aides peuvent être conseillées afin d'améliorer le quotidien des patients arthrosiques, telles qu'une canne de marche (simple avec une poignée en T, en équerre ou en arc de cercle ; canne anglaise avec un contre-appui au dos de l'avant-bras, permettant de s'appuyer beaucoup plus ; tripode, quadripode ou canne pliante télescopique...) ou des outils permettant de saisir les objets et d'éviter ainsi de se baisser. Dans les formes très invalidantes, l'adaptation du logement doit parfois également être envisagée.

\section{Note}

${ }^{1}$ La posologie maximale de 4 grammes par jour ne devrait être proposée que sur prescription médicale et non au cours d'un conseil officinal.

\section{Références}

[1] Guglielmo D, Hootman JM, Murphy LB et al. Health care provider counseling for weight loss among adults with arthritis and overweight or obesity - United States, 2002-2014. MMWR Morb Mortal Wkly Rep. 2018;67(17):485-90.

[2] Association française de lutte antirhumatismale (Aflar). www.aflar.org/l-arthrose

[3] Aigner T, Stöve J. Collagens-major component of the physiological cartilage matrix, major target of cartilage degeneration, major tool in cartilage repair. Adv Drug Deliv Rev. 2003;55(12):1569-93.

[4] Haute Autorité de santé (HAS). Prise en charge de l'arthrose : le paracétamol en première intention lors des crises douloureuses. www.has-sante.fr/portail/upload/docs/application/pdf/201402/focus_arthrose_mars_2014_bat_ce.pdf 
[5] Agence nationale de sécurité du médicament et des produits de santé (ANSM). Jamais d'AINS à partir du début du 6ème mois de grossesse - Point d'Information. 26 Janvier 2017.

https://ansm.sante.fr/S-informer/Points-d-information-Points-d-information/Rappel-Jamais-d-AINSa-partir-du-debut-du-6eme-mois-de-grossesse-Point-d-Information

Déclaration de liens d'intérêts

L'auteur déclare ne pas avoir de liens d'intérêts.

\section{Illustration}

\section{Cle_illus1}

(C) Laszlo/stock.adobe.com

L'exercice physique est bénéfique pour limiter le risque d'arthrose et son évolution à condition de s'échauffer et d'acquérir de bons gestes techniques.

Sur 1 colonne + marge (ou 1 colonne) en deuxième page en haut 